Provided for non-commercial research and education use. Not for reproduction, distribution or commercial use.

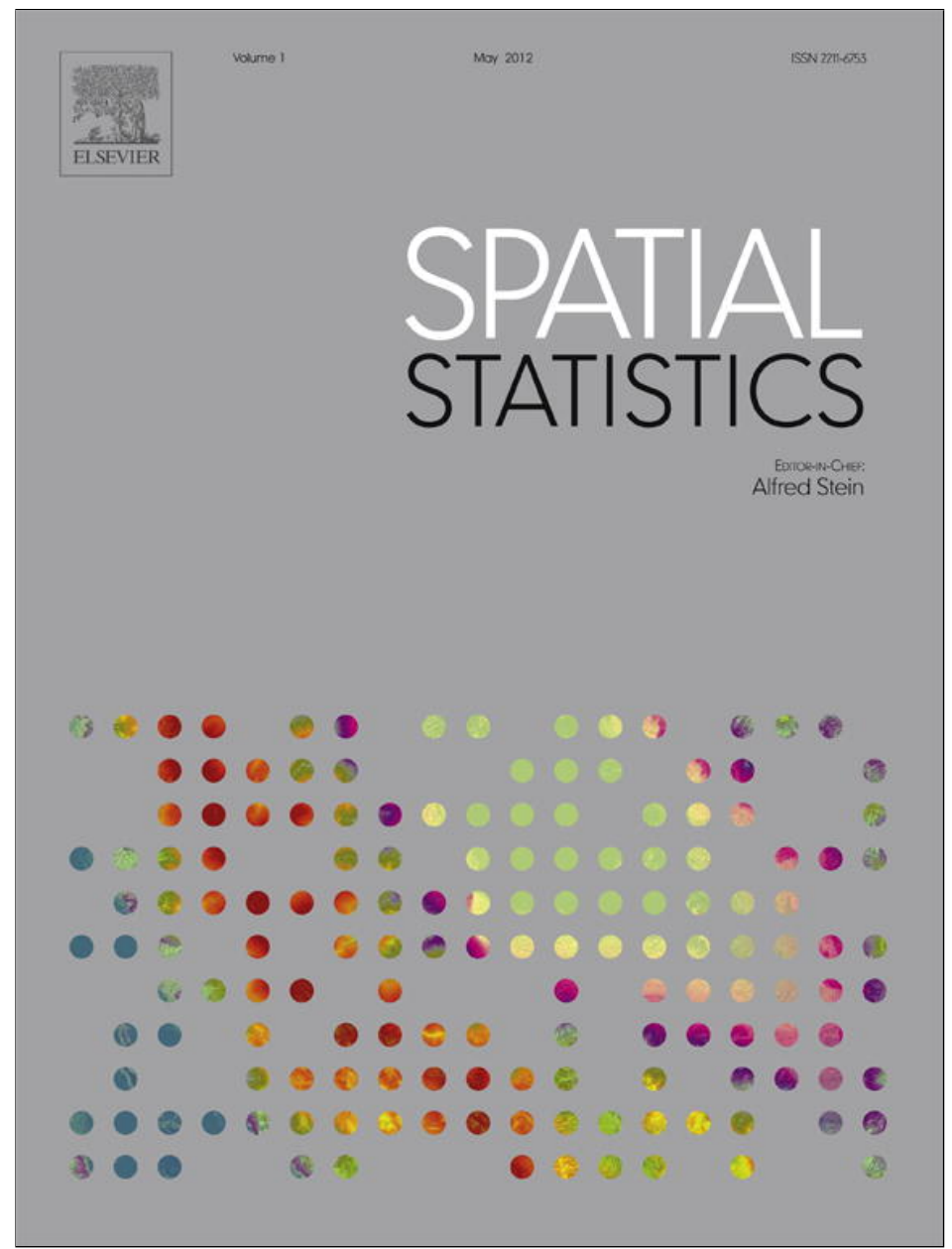

This article appeared in a journal published by Elsevier. The attached copy is furnished to the author for internal non-commercial research and education use, including for instruction at the authors institution and sharing with colleagues.

Other uses, including reproduction and distribution, or selling or licensing copies, or posting to personal, institutional or third party websites are prohibited.

In most cases authors are permitted to post their version of the article (e.g. in Word or Tex form) to their personal website or institutional repository. Authors requiring further information regarding Elsevier's archiving and manuscript policies are encouraged to visit:

http://www.elsevier.com/copyright 


\title{
An introduction to planar random tessellation models
}

\author{
M.N.M. van Lieshout \\ CWI, Amsterdam, The Netherlands
}

\section{A R T I C L E I N F O}

\section{Article history:}

Received 3 January 2012

Accepted 27 February 2012

Available online 10 March 2012

\section{Keywords:}

Arak-Clifford-Surgailis polygonal Markov field

Isotropic Poisson line process

Johnson-Mehl tessellation

Laguerre tessellation

STIT model

Voronoi tessellation

\begin{abstract}
A B S T R A C T
The goal of this paper is to give an overview of random tessellation models. We discuss the classic isotropic Poisson line tessellation in some detail and then move on to more complicated models, including Arak-Clifford-Surgailis polygonal Markov fields and their Gibbs field counterparts, crystal growth models such as the Poisson-Voronoi, Johnson-Mehl and Laguerre random tessellations, and the STIT nesting scheme. An extensive list of references is included as a guide to the literature.
\end{abstract}

(c) 2012 Elsevier B.V. All rights reserved.

\section{Introduction}

Random tessellations, also known as random mosaics or stochastic networks, are random partitions of the plane into disjoint regions. Mosaics arise naturally in many contexts. Examples include tilings, crystals, cellular structures, land use maps, galaxies, communication networks, crack patterns, foams, and so on. The use of tessellations has a long history in the geosciences-both as models in their own right and as spatial interpolation tools. See for example Thiessen's classic paper (Thiessen, 1911) on estimating regional rainfall or Harding's work (Harding, 1923) on the estimation of ore reserves. More recent work on related problems includes (Ju et al., 2011; Møller and Skare, 2001). Note that supporting data structures are routinely implemented in GIS systems (Rigaux et al., 2001).

Random tessellations are at the heart of stochastic geometry, the branch of mathematics that concerns itself with modelling and analysing complicated geometrical structures. The aim of this paper is to introduce this fascinating subject to the non-expert and to provide pointers to the literature. For simplicity, all models are described in the plane, but similar models exist in three dimensions.

Formally, a planar tessellation is a collection of mutually disjoint open sets $\left\{C_{1}, C_{2}, \ldots\right\}, C_{i} \subset \mathbb{R}^{2}$, such that:

E-mail address: Marie-Colette.van.Lieshout@cwi.nl. 


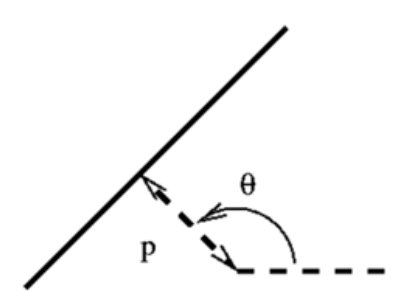

Fig. 1. Parametrisation of lines.

- $C_{i} \cap C_{j}=\emptyset$ for $i \neq j$;

- $\cup_{i} \bar{C}_{i}=\mathbb{R}^{2}$;

- for any bounded set $B \subset \mathbb{R}^{2}$, the set $\left\{i: C_{i} \cap B \neq \emptyset\right\}$ is finite.

Here $\bar{C}$ denotes the topological closure of $C$. In words, the 'tiles' $\bar{C}_{i}$ fill the plane, their interiors do not overlap, and only finitely many of them are needed to cover a bounded region. Additional assumptions can be imposed, for example that the sets are non-empty, convex, bounded, or polygons. The $C_{i}$ are called the cells of the tessellation. Examples are shown in Figs. 2 and 4-6.

Randomness can be generated in a number of ways. For example, one may draw a number of random lines and use them to delineate the boundaries of polygonal cells. Alternatively, a set of points may be generated from which regions or lines are grown until hit by other regions or lines. A pattern of lines may also be used as a skeleton on which to draw non-convex polygonal shapes. Furthermore, operations such as superposition and partitioning can be applied to the cells of a tessellation, either once or as part of an iterative scheme. Most of these mechanisms are briefly discussed in Chapters 10 (sic) of the textbooks (Stoyan et al., 1995; Schneider and Weil, 2008) on stochastic geometry, the lecture notes (Møller, 1994) and the monograph (Okabe et al., 2000) focus on region growing. From a historical perspective the charming booklet (Kendall and Moran, 1963), in summarising classic theory on 'uniformly' distributed random geometrical objects and raising a number of open problems, stimulated research. A partial review of recent developments can be found in Calka (2010).

The plan of this paper is as follows. We first consider Poisson line tessellations in Section 2, then move on to Arak-Clifford-Surgailis polygonal field models in Section 3. Ongoing research on discrete polygonal fields that promise to be useful for image classification and segmentation is touched upon. In Section 4.1, we describe crystal growth models including random Voronoi, Johnson-Mehl and Laguerre tessellations. The new class of stationary iteration stable random tessellations is discussed in Section 4.2. The paper closes with a summary and conclusion.

\section{The isotropic Poisson line tessellation}

The isotropic Poisson line tessellation is one of the fundamental models in stochastic geometry. In order to describe it, recall that a straight line in the plane can be parametrised by the signed length and orientation of the perpendicular joining the origin with the line; cf. Fig. 1. More specifically,

$$
l_{\theta, p}=\left\{(x, y) \in \mathbb{R}^{2}: x \cos \theta+y \sin \theta=p\right\}
$$

for $\theta \in[0, \pi), p \in \mathbb{R}$. The measure $\mu$ defined by

$$
\mu(E)=\int_{E} d \theta d p
$$

for $E \subseteq[0, \pi) \times \mathbb{R}$ is the unique measure up to a scalar factor that is invariant under rigid motions, that is,

$$
\mu(f(E))=\mu(E)
$$

for all $f$ that are compositions of translations and rotations (Poincaré, 1912).

Definition 1. The isotropic Poisson line process $L$ on $\mathbb{R}^{2}$ with rate $\lambda>0$ is a Poisson process on $[0, \pi) \times \mathbb{R}$ with intensity measure $\lambda \mu$. 


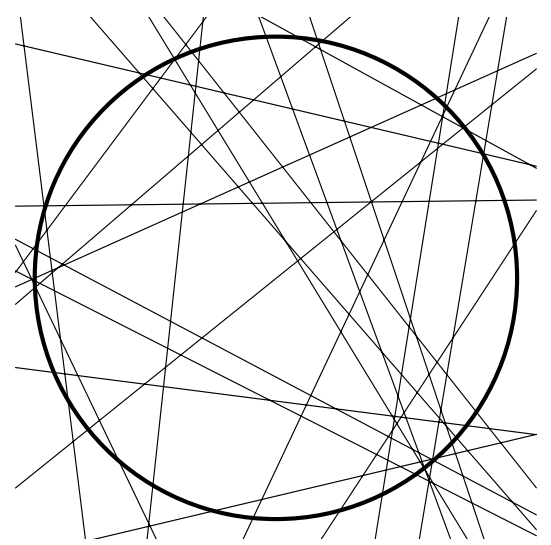

Fig. 2. Sample of a unit-rate isotropic Poisson line process hitting a ball of radius 3 .

In Euclidean coordinates, the heads of the perpendiculars that join the lines with the origin form a Poisson point process on $\mathbb{R}^{2}$ with intensity function $\lambda /\|(x, y)\|$. It is important to note that the intensity decreases as a function of the distance to the origin, a property that ensures that no bounded set is hit by infinitely many lines coming from far away.

The line process $L$ (Definition 1 ) inherits from the underlying Poisson process a number of desirable properties. First, almost surely $L$ consists of countably many lines but every bounded set $B \subset \mathbb{R}^{2}$ is hit by finitely many lines only. We shall write $[B] \subset[0, \pi) \times \mathbb{R}$ for the hitting set of $B$, that is $[B]=\left\{(\theta, p): l_{\theta, p} \cap B \neq \emptyset\right\}$. Conditionally on the event that $[B]$ contains $n$ lines, these lines are independent and identically distributed according to the probability density $\mu(\cdot \cap[B]) / \mu([B])$. Finally, almost surely no lines of $L$ share the same orientation and no triple of lines intersect in a common point.

Example 1. Let $B=\left\{(x, y) \in \mathbb{R}^{2}:\|(x, y)\| \leq r\right\}$ be the ball of radius $r$. Then a line $l_{\theta, p}$ hits $B$ if and only if $-r \leq p \leq r$, so

$$
\lambda \mu([B])=\lambda \int_{0}^{\pi} \int_{-r}^{r} d \theta d p=2 \lambda \pi r .
$$

A similar reasoning shows that conditionally on $n$ lines hitting the ball, their parameters are independent and identically distributed with probability density $1 /(2 \pi r)$ on $[0, \pi) \times[-r, r]$; cf. Miles (1964). A realisation of $L \cap[B]$ for $\lambda=1$ and $r=3$ is given in Fig. 2 .

Several equivalent definitions of an isotropic Poisson line process exist. An immediate consequence of Definition 1 is that the signed distances to the origin form a Poisson process with rate $\lambda \pi$ on the line, and their orientations are independent and uniformly distributed on $[0, \pi)$ (Miles, 1964). Thus, one may construct the process by generating a Poisson process of radii on $\mathbb{R}^{+}$with rate $2 \lambda \pi$ and, conditional on these radii $\rho_{i}$, draw independent lines tangent to the corresponding discs $\partial B\left(0, \rho_{i}\right)$ at uniformly chosen locations.

Another definition is based on line transects (Wolfowitz, 1949; Miles, 1964).

Theorem 1. Let $\ell$ be a fixed line and $L$ an isotropic Poisson line process. Then the intersections of the lines parametrised by $L$ with $\ell$ form a Poisson point process on $\ell$ with rate $2 \lambda$ and the intersection angles made with the line are independent and identically distributed with probability density $\frac{1}{2} \sin \theta$ on $[0, \pi)$.

Note that Theorem 1 immediately leads to a third construction of $L$. Since the lines of $L$ are mutually independent, one may replace the fixed line $\ell$ with one from $L$.

The isotropic Poisson line process induces a random tessellation whose cells are almost surely nonempty convex polygons. In order to describe its distribution, one may consider the cell that contains the origin. This cell is almost surely uniquely defined and known as the Crofton cell. Due to the fact that large cells are more likely to contain the origin, the Crofton cell is larger than a 'typical' cell defined through the Palm measure (Mecke, 1967; Møller, 1989). More specifically, assign to each polygon $C$ in the random tessellation induced by the isotropic Poisson line process $L$ a unique centroid $z(C)$, for 

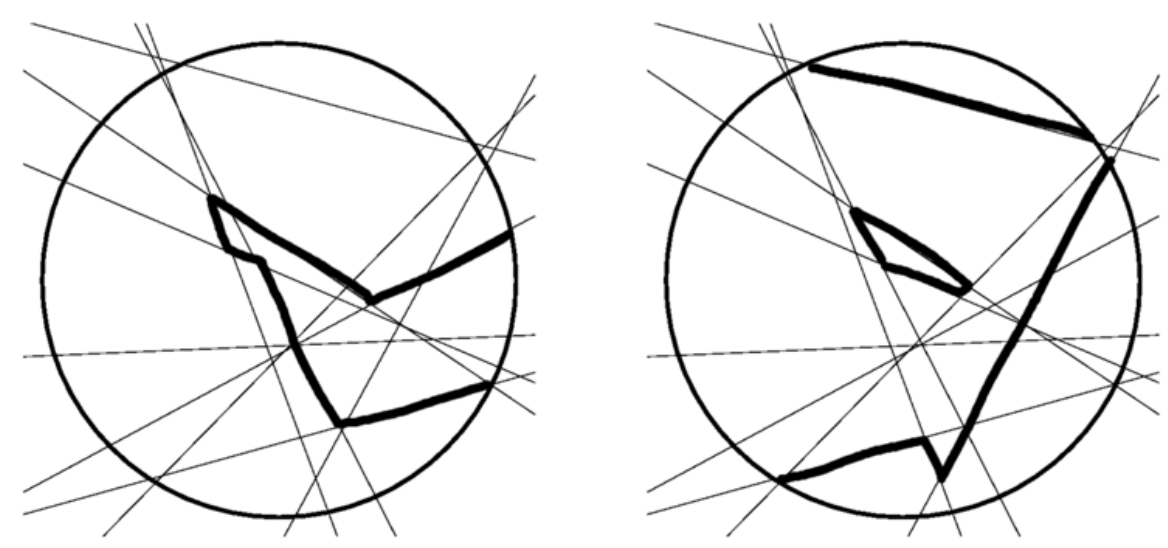

Fig. 3. Two polygonal configurations (thick lines) drawn on the same skeleton (thin lines).

example the centre of the largest ball included in the polygon. Since $L$ is stationary, so is the centroid process. Its rate is equal to $\beta=\pi \lambda^{2}$; see Stoyan et al. (1995, Chapter 10) for further details.

Definition 2. Let $L$ be an isotropic Poisson line process with rate $\lambda$ and $B$ a set with finite area $|B|>0$. The typical polygon $P_{L}$ of the induced random tessellation satisfies the set of equations

$$
\mathbb{E} f\left(P_{L}\right)=\frac{1}{\beta|B|} \mathbb{E}\left[\sum_{C: z(C) \in B} f(C-z(C))\right]
$$

for all integrable real-valued functions $f$.

The definition does not depend on the choice of $B$ (Møller, 1989). Intuitively speaking, each centroid falling in some set $B$ is taken as the origin and the function values of the thus shifted polygons averaged to yield the Palm function value. Since (1) must hold for all $f$, the distribution of $P_{L}$ is uniquely specified.

Partial results on the distribution of $P_{L}$ are available. For example the distribution of the diameter of the largest ball contained in $P_{L}$ is exponentially distributed (Miles, 1964).

Example 2. For a non-empty convex polygon $C$, write $d(C)$ for the diameter of the largest ball contained in $C$ and $z(C)$ for the centre of this ball. Set $f(C)=1\{d(C)>r\}$. Note that $f(C)=1$ if and only if no line parametrised by a member of $L$ hits the closed ball centred at $z(C)$ with radius $r / 2$. By Example 1, the probability of this event is $\exp (-\lambda \pi r)$. Consequently, $d\left(P_{L}\right)$ is exponentially distributed with parameter $\lambda \pi$.

For a recent overview of further results in this direction, see Calka (2010).

\section{Arak polygonal Markov field models}

In Section 2, we considered the isotropic Poisson line process $L$ and saw that its induced tessellation consists of convex polygons. The goal of this section is to discuss a class of models that relaxes the convexity assumption while keeping some of the properties of $L$.

The idea is to use the Poisson lines as a skeleton to define polygonal fields. Each line cannot be used more than once, but many tessellations can be built on a single realisation of $L$. An illustration is given in Fig. 3.

Formally, let $D$ be a bounded, convex open subset of the plane. The family $\Gamma_{D}$ of admissible polygonal configurations in $D$ is the set of planar graphs $\gamma$ in $\bar{D}=D \cup \partial D$ with non-intersecting line segments as edges such that no two edges are collinear, all vertices in $D$ have degree 2 , and the vertices on the boundary $\partial D$ are of degree 1 (Arak, 1982; Arak and Surgailis, 1989).

Definition 3. Let $L$ be a unit-rate Poisson line process on the hitting set of $D$ and write $\Gamma_{D}(L)$ for the family of $\gamma \in \Gamma_{D}$ such that $\gamma$ is a subset of the lines parametrised by $L$ and for each such line $l_{i}, l_{i} \cap \gamma$ 


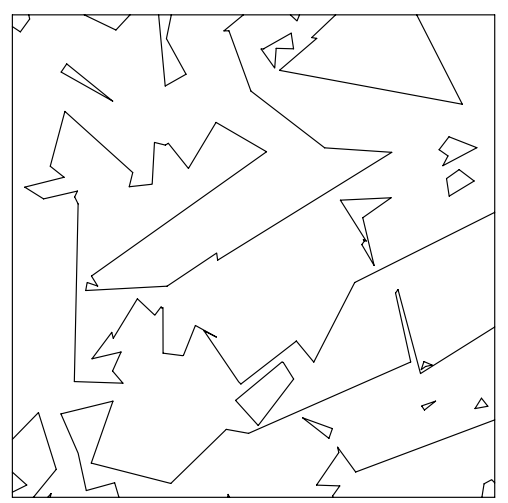

Fig. 4. Sample of an Arak polygonal Markov field in a square of side length 3.

consists of exactly one non-zero length interval. The Arak polygonal Markov field $\mathcal{A}_{D}$ on $D$ is defined by

$$
\mathbb{P}\left(\mathcal{A}_{D} \in G\right)=\frac{1}{Z} \mathbb{E}\left[\sum_{\gamma \in \Gamma_{D}(L) \cap G} \exp (-2 l(\gamma))\right]
$$

for $G \subseteq \Gamma_{D}$. Here $l(\gamma)$ denotes the total edge length of $\gamma$ and the expectation is with respect to the distribution of $L$.

A realisation of $\mathcal{A}_{D}$ for $D=(0,3) \times(0,3)$ is given in Fig. 4. Note that $\mathcal{A}_{D}$ forms a tessellation of polygons that are not necessarily convex that may be nested.

Like the isotropic Poisson line process $L$ on which it is built, the Arak polygonal Markov field is isotropic. It shares a number of other properties with $L$.

Theorem 2. - Consistency: $\mathcal{A}_{D}$ is equal in distribution to the restriction of $\mathcal{A}_{D^{\prime}}$ to $D$ for $D^{\prime} \supseteq D$.

- Poisson line transects: for a given straight line $\ell, \mathcal{A}_{D} \cap \ell$ is a Poisson point process on $\ell$ with rate 2 .

- Solvability: the normalising constant $Z$ in Definition 3 is available in closed form and equal to $\exp [\pi|D|]$.

The extra term exp $[l(\partial D)]$ in Arak and Surgailis (1989, Theorem 4.1) is due to the fact that in that paper the model is defined with respect to the unnormalised isotropic Poisson line measure.

The proof of Theorem 2 is based on a dynamic representation (Arak and Surgailis, 1989), the main idea of which is to interpret the polygonal boundaries of the field as the traces left by particles travelling in two-dimensional time-space. In other words, coordinates $(t, y) \in D$ are interpreted as the one-dimensional spatial location $y$ of a particle at time $t$. Its progression is Markovian, explaining the nomenclature. For simplicity, assume that $D$ is a rectangle.

In $D$, particles are born according to a Poisson point process with rate $\pi$. On the boundary $\partial D$, births occur according to a Poisson point process with rate 2 on the left side; on the top and bottom sides the constraint that particles must move forward in time reduces the rate to 1 . Each birth site in $D$ emits two particles, so its degree is 2 as required. The angle between the initial particle traces has probability density $(\sin \theta) / 2$. Each boundary birth site emits a single particle with the same probability density for the angle between its trace and the border conditional on moving forward in time; cf. Theorems 1 and 2.

All particles evolve independently in time according to the following rules. Each particle moves with constant velocity for an exponentially distributed distance (rate 2), after which it changes velocity in such a way that the angle between its old and new traces in $D$ has probability density $(\sin \theta) / 2$, in accordance with the line transect property. In the case of a collision between two particles, that is, equal spatial coordinates $y$ at some time $t$, both of them die; when a particle hits $\partial D$, its trace terminates there and then.

The model of Definition 3 is not the only consistent polygonal Markov field satisfying appealing properties similar to those outlined in Theorem 2. A characterisation of the full class seems to be an 


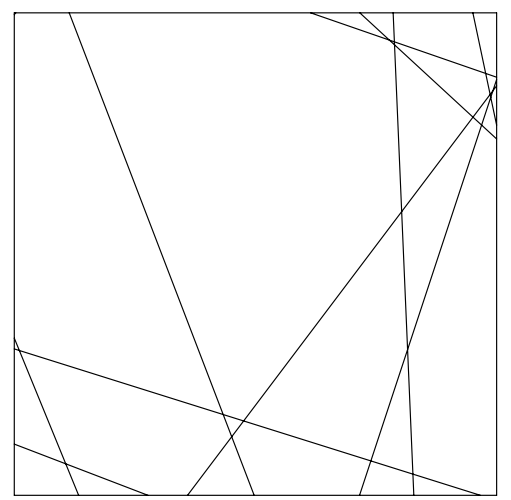

Fig. 5. Sample of a polygonal Markov field with interior vertices of degree 4 in a square of side length 3.

open problem, but a large family is presented in Arak et al. (1993) and Arak and Surgailis (1989). Below, we give an example of a field exhibiting only vertices of degree 4 in $D$. For a model with vertices of degree 3 only, the reader is referred to Miles and Mackisack (2002).

Example 3. In the dynamic representation described above, suppose that no births occur in $D$ and a particle entering at $\partial D$ sticks to its initial velocity until leaving $D$. Furthermore, suppose that upon a collision of two particles, they both survive and continue their trajectory. The resulting process satisfies the properties of Theorem 2 . Note that its distribution coincides with that of the unit-rate Poisson line process on $[D \cup \partial D]$. A realisation for $D=(0,3) \times(0,3)$ is given in Fig. 5 .

Consistent polygonal Markov field models with both internal vertices of degree 2 and ones of degree 4 can be defined in a straightforward fashion. Simply let colliding particles die with probability $p_{V}$ in the dynamic representation and survive with the complementary probability $p_{X}=1-p_{V}$. In general, the distribution of the typical polygon in consistent polygonal Markov field models seems difficult to obtain. Partial results can be found in Schreiber $(2005,2008)$.

To conclude this section, we describe polygonal Markov fields drawn on a fixed collection of lines $\mathcal{T}$, for example a regular planar lattice (Schreiber and Lieshout, 2010). We assume that every bounded set $B \subset \mathbb{R}^{2}$ is hit by finitely many lines and no triple of lines intersect in a common point. Furthermore, no lines may intersect on the boundary $\partial D$ of $D$ and there are no segments of $\partial D$ along lines of $\mathcal{T}$.

The family $\Gamma_{D}(\mathcal{T})$ of admissible polygonal configurations in $D$ built on $\mathcal{T}$ is the set of planar graphs $\gamma$ in $\bar{D}=D \cup \partial D$ such that all edges of $\gamma$ lie on lines of $\mathcal{T}$, all vertices in $D$ have degree 2 , and the vertices on $\partial D$ are of degree 1 . Note that edges may be collinear!

Example 4. Consider the following dynamic representation. Choose time-space birth sites independently with probabilities:

- $p^{2}$ at each intersection point of two lines of $\mathcal{T}$;

- $p /(1+p)$ at each intersection of a line of $\mathcal{T}$ with $\partial D$;

for some $p \in(0,1)$. Each birth site in $D$ emits two particles unless some previously born particle hits the site in which case the birth does not occur; each birth site on the boundary emits one particle. The initial traces of the emitted particles lie along the lines of $\mathcal{T}$ in the direction of time. All particles evolve independently in time as follows. When a particle moving along some line $l_{1}$ reaches a point of intersection with another line, say $l_{2}$, it changes its direction and continues along $l_{2}$ with probability $p$ and keeps moving along $l_{1}$ with the complementary probability $1-p$. As in the continuous case, particles die upon collisions.

The model described in Example 4 is consistent and properties similar to those outlined in Theorem 2 hold. In particular, the random set of intersections with a given straight line $\ell$ that does not pass through any intersection point of $\mathcal{T}$ is equal in distribution to the combined intersections with $\ell$ of the random subset of $\mathcal{T}$ obtained by selecting each line with probability $p /(1+p)$ independently of the others (Schreiber and Lieshout, 2010). A typical realisation on a regular lattice with $p=0.5$ is given in Fig. 6. Furthermore, Example 4 can be generalised to allow for interior vertices of degrees 3 and 4 as well as 2 (Lieshout, 2012). 


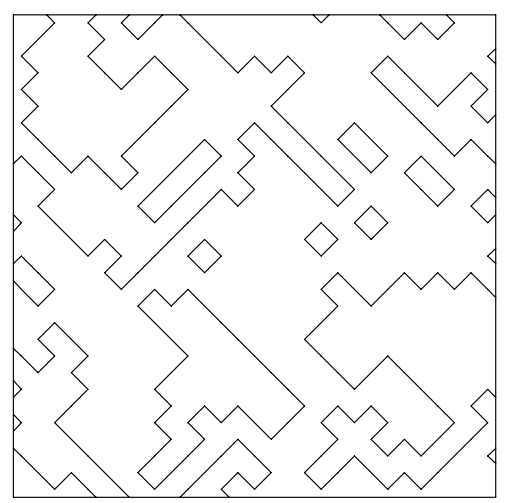

Fig. 6. Sample of a polygonal field (Example 4) on a regular lattice with $p=0.5$.

\section{Other random tessellation models}

\subsection{Crystal growth models}

The models described in Sections 2 and 3 are based on lines. In this section, we shall describe models that are based on region growing from a given set of centres or nuclei.

Let $\left\{x_{1}, x_{2}, \ldots\right\}$ be a collection of points in $\mathbb{R}^{2}$. We assume that every bounded set contains finitely many points, no four points are located on the boundary of a disc, and no three points are collinear. Realisations of stationary planar Poisson point processes, for instance, almost surely possess these properties.

Definition 4. The Voronoi cell of $x_{i}$ in $\left\{x_{1}, x_{2}, \ldots\right\}$ is the set

$$
\left\{y \in \mathbb{R}^{2}:\left\|x_{i}-y\right\| \leq\left\|x_{j}-y\right\| \quad \forall j \neq i\right\}
$$

of points that are at least as close to $x_{i}$ as to any other point.

Since the cells cover the plane, their interiors form a tessellation. The dual triangulation that arises by placing edges between those $x_{i}$ and $x_{j}$ that share a common Voronoi border is known as the Delaunay tessellation (Okabe et al., 2000).

To see how a Voronoi tessellation can be interpreted as a growth model, suppose that nuclei are placed at each of the $x_{i}$ and start growing simultaneously at a constant rate in all directions to form crystals; when a growing crystal meets another growing crystal in a certain direction, the growth stops, but it may continue in other directions until stopped by meeting another growing crystal. The crystals formed in this way are convex but may be unbounded, for example if the collection of nuclei is finite. If all crystals are bounded, they are convex polygons.

The only analytically tractable case seems to be that in which the nuclei are generated according to a stationary Poisson point process with rate $\lambda>0$. By the Slivnyak-Mecke theorem, the Palm distribution of a Poisson point process amounts to adding an extra point to it. Hence we have the following result.

Theorem 3. The typical polygon of a Voronoi tessellation generated by a stationary Poisson point process $X$ with rate $\lambda$ coincides in distribution with the polygon in the Voronoi tessellation generated by $X \cup\{0\}$ that contains 0 . Its mean area is $1 / \lambda$, the mean number of vertices is 6 and the mean perimeter is $4 \lambda^{-1 / 2}$.

The mean values are due to Meijering (1953) and Miles (1970). For more details, see Møller (1994, Chapter 4) or Calka (2010, Section 5.2) and references therein.

Other crystal growth models can be defined by relaxing the assumptions. For example, allowing the nuclei to start growing at different times leads to the Johnson-Mehl tessellation. Note that in this case the crystals may be empty and not necessarily convex. Alternatively, assume that the growth rates may vary from crystal to crystal or depend on the direction. 
Example 5. Let $\left\{\left(x_{1}, r_{1}\right),\left(x_{2}, r_{2}\right), \ldots\right\}$ be a collection of points $x_{i}$ with associated marks $r_{i}>0$ and define the Laguerre cell of $x_{i}$ by

$$
\left\{y \in \mathbb{R}^{2}:\left\|y-x_{i}\right\|^{2}-r_{i}^{2} \leq\left\|y-x_{j}\right\|^{2}-r_{j}^{2} \quad \forall j \neq i\right\} .
$$

Thus the Euclidean distance used in the definition of the Voronoi cell (Definition 4) is replaced by the power distance $\left\|y-x_{i}\right\|^{2}-r_{i}^{2}$. The Laguerre cells may be empty. If not, they are convex polygons. Note that the nucleus does not necessarily lie in its own cell.

As for Voronoi tessellations, Laguerre tessellations are most tractable if the underlying set of points is generated by an independently marked stationary Poisson point process. Conditions have to be imposed on the mark distribution for the process to be well-defined. Analytic results can be found in Lautensack and Zuyev (2008), including integral formulae for the mean values of the typical cell in the spirit of Theorem 3.

\subsection{Iterated tessellation models}

The last class of tessellation models that we describe is based on the concept of nesting. Suppose a random tessellation $Y$ is given and has cells $C_{i}$ (cf. Section 1). Moreover, suppose that for every cell we have a random tessellation $Y_{i}$, and assume that the sequence $Y_{i}, i \in \mathbb{N}$, is independent and identically distributed. Then a new tessellation $\tilde{Y}=\tilde{Y}\left(Y, Y_{1}, Y_{2}, \ldots\right)$ can be obtained by subdividing each $C_{i}$ through intersecting it with the cells of $Y_{i}$ (Stoyan et al., 1995).

Example 6. Let $Y$ be an isotropic Poisson line process and suppose that the $Y_{i}$ are also isotropic Poisson line processes, independent of $Y$, possibly with a different rate. Then the intersections of the $Y_{i}$ with the cells of $Y$ form a nested Poisson line process. Further examples, sufficient conditions for stationarity as well as mean value results are presented in Maier and Schmidt (2003).

The nesting operation may be iterated and combined with appropriate rescaling. Nagel and Weiss (2003) showed that such repeated nesting leads to a stationary limit tessellation if and only if the limit model is stable with respect to iteration (abbreviated STIT). In a follow-up paper (Nagel and Weiss, 2005) a characterisation and construction of the STIT tessellations in compact windows $W$ of nonempty interior is given. Below we give the construction using the invariant measure $\mu$ introduced in Section 2. As before, $[W]$ denotes the hitting set of $W$.

Definition 5. Suppose that $W$ has an exponentially distributed lifetime with mean $1 / \mu([W])$, after which a line appears according to $\mu(\cdot \cap[W]) / \mu([W])$ which splits $W$ into two parts, $W_{1}$ and $W_{2}$. In their turn, each $W_{i}$ lives for an exponentially distributed time with mean $1 / \mu\left(\left[W_{i}\right]\right), i=1,2$, after which it is divided by a random line as before, and so on. The iteration is terminated after some fixed time has elapsed. Note that smaller cells tend to live longer than larger ones.

The output of the construction described in Definition 5 is a random tessellation of $W$ into convex polygons, possibly chopped off by the boundary of $W$.

Theorem 4. The construction in Definition 5 is well-defined, almost surely contains a finite number of cells and is consistent.

Note that the degree of the vertices in the interior of $W$ is 3 . In this sense, the model is similar to models in Miles and Mackisack (2002), but the latter are not STIT. The typical cell distribution for both models coincides with that for the isotropic Poisson line process described in Section 2, even though the vertices in the latter model all have degree 4 ! Geometric characteristics of STIT tessellations are discussed in a series of preprints by Schreiber and Thäle (2010). 


\section{Discussion}

In this paper, we endeavoured to give an introduction to the field of random tessellations for the non-expert. We described in detail various equivalent constructions of the fundamental isotropic Poisson line process. We then moved on to the lesser known class of polygonal Markov fields and included sampled realisations to illustrate the variety in mosaics that can be obtained. We then discussed crystal growth models, and concluded our overview by presenting the relatively recently discovered class of random tessellations that are stable with respect to repeated nesting.

Most textbooks (Møller, 1994; Okabe et al., 2000; Schneider and Weil, 2008; Stoyan et al., 1995) concentrate on the classic random Voronoi and Poisson line process tessellations. Our focus has been more on skeleton models, reflecting our current research interest in tessellation based image segmentation. The idea of using polygonal Markov field models for this purpose can be traced back to Clifford and Middleton (1989); see also Clifford and Nicholls (1994). Since the Monte Carlo methods employed at that time turned out to be rather onerous, the theme was not picked up again until the mid-2000s (Paskin and Thrun, 2005) when further theoretical results (Schreiber, 2005) motivated the development of conceptually and computationally easier algorithms (Kluszczyński et al., 2005, 2007; Schreiber and Lieshout, 2010; Lieshout, 2012). In the meantime, Voronoi (Green, 1995; Heikkinen and Arjas, 1998; Møller and Skare, 2001) and triangulation (Nicholls, 1998) models had also been tried.

Recent years have seen renewed interest in random tessellation models, especially as regards the distribution of the typical cell, interactions between neighbouring cells and asymptotics. It is hoped that the introduction and overview given in this paper will help to motivate further research.

\section{Acknowledgements}

This research was supported by The Netherlands Organisation for Scientific Research NWO (613.000.809). The author would like to thank K. Kayabol for a careful reading of the draft.

\section{References}

Arak, T., 1982. On Markovian random fields with finite number of values. 4th USSR-Japan symposium on probability theory and mathematical statistics, Abstracts of Communications. Tbilisi.

Arak, T., Clifford, P., Surgailis, D., 1993. Point-based polygonal models for random graphs. Advances in Applied Probability 25, 348-372.

Arak, T., Surgailis, D., 1989. Markov fields with polygonal realisations. Probability Theory and Related Fields 80, 543-579.

Calka, P., 2010. Tessellations. In: Kendall, W.S., Molchanov, I. (Eds.), New Perspectives in Stochastic Geometry. Oxford University Press, pp. 145-169.

Clifford, P., Middleton, R.D., 1989. Reconstruction of polygonal images. Journal of Applied Statistics 16, 409-422.

Clifford, P., Nicholls, G.K., 1994. A Metropolis sampler for polygonal image reconstruction. Electronic version available at: http://www.stats.ox.ac.uk/ clifford/papers/met_poly.html.

Green, P.J., 1995. Reversible jump Markov chain Monte Carlo computation and Bayesian model determination. Biometrika 82, 711-732.

Harding, J.E., 1923. How to calculate tonnage and grade of an ore body. Engineering and Mining Journal Press 116, 445-448.

Heikkinen, J., Arjas, E., 1998. Non-parametric Bayesian estimation of a spatial Poisson intensity. Scandinavian Journal of Statistics $25,435-450$

Ju, L., Ringler, T., Gunzburger, M., 2011. Voronoi tessellations and their application to climate and global modeling. Lecture Notes in Computational Science and Engineering 80, 313-342.

Kendall, M.G., Moran, P.A.P., 1963. Geometrical Probability. Griffin, London.

Kluszczyński, R., Lieshout, M.N.M. van, Schreiber, T., 2005. An algorithm for binary image segmentation using polygonal Markov fields. In: F. Roli and S. Vitulano (Eds.), Image Analysis and Processing, Proceedings of the 13th International Conference on Image Analysis and Processing. Lecture Notes in Computer Science 3615, 383-390.

Kluszczyński, R., Lieshout, M.N.M. van, Schreiber, T., 2007. Image segmentation by polygonal Markov fields. Annals of the Institute of Statistical Mathematics 59, 465-486.

Lautensack, C., Zuyev, S., 2008. Random Laguerre tessellations. Advances in Applied Probability 40, 630-650.

Lieshout, M.N.M. van, 2012. Multi-colour planar Markov fields. CWI, Amsterdam. Manuscript.

Maier, R., Schmidt, V., 2003. Stationary iterated tessellations. Advances in Applied Probability 35, 337-353.

Mecke, J., 1967. Stationäre zufällige Maße auf lokalkompakten Abelschen Gruppen. Zeitschrift für Wahrscheinlichkeitstheorie und verwandte Gebiete 9, 36-58.

Meijering, J.L., 1953. Interface area, edge length, and number of vertices in crystal aggregates with random nucleation. Philips Research Reports 8, 270-290.

Miles, R.E., 1964. Random polygons determined by random lines in a plane. Proceedings of the National Academy of Sciences USA 52, 901-907. 
Miles, R.E., 1970. On the homogeneous planar Poisson point process. Mathematical Biosciences 6, 85-127.

Miles, R.E., Mackisack, M.S., 2002. A large class of tessellations with the classic Poisson polygon distributions. Forma 17, 1-17. Møller, J., 1989. Random tessellations in $\mathbb{R}^{d}$. Advances in Applied Probability 21, 37-73.

Møller, J., 1994. Lectures on Random Voronoi Tessellations. In: Lecture Notes in Statistics, vol. 87. Springer.

Møller, J., Skare, Ø., 2001. Bayesian image analysis with coloured Voronoi tessellations and a view to applications in reservoir modelling. Statistical Modelling 1, 213-232.

Nagel, W., Weiss, V., 2003. Limits of sequences of stationary planar tessellations. Advances in Applied Probability 35, $123-138$.

Nagel, W., Weiss, V., 2005. Crack STIT tessellations: characterization of stationary random tessellations stable with respect to iteration. Advances in Applied Probability 37, 859-883.

Nicholls, G.K., 1998. Bayesian image analysis with Markov chain Monte Carlo and coloured continuum triangulation models. Journal of the Royal Statistical Society, Series B 60, 643-659.

Okabe, A., Boots, B., Sugihara, K., Chiu, S.N., 2000. Spatial Tessellations: Concepts and Applications of Voronoi Diagrams, second ed. Wiley.

Paskin, M.A., Thrun, S., 2005. Robotic mapping with polygonal random fields. Proceedings in Artificial Intelligence UAI-05.

Poincaré, H., 1912. Calcul des probabilités, second ed., Paris.

Rigaux, P., Scholl, M., Voisard, A., 2001. Spatial Databases With Application to GIS. Elsevier.

Schneider, R., Weil, W., 2008. Stochastic and Integral Geometry. Springer.

Schreiber, T., 2005. Random dynamics and thermodynamic limits for polygonal Markov fields in the plane. Advances in Applied Probability 37, 884-907.

Schreiber, T., 2008. Non-homogeneous polygonal Markov fields in the plane: graphical constructions and geometry of higherorder correlations. Journal of Statistical Physics 132, 669-705.

Schreiber, T., Lieshout, M.N.M. van, 2010. Disagreement loop and path creation/annihilation algorithms for binary planar Markov fields with applications to image segmentation. Scandinavian Journal of Statistics 37, 264-285.

Schreiber, T., Thäle, C., 2010. Typical geometry, second-order properties and central limit theory for iteration stable tessellations. Electronic version available at: http://arxiv.org/abs/1001.0990v3.

Stoyan, D., Kendall, W.S., Mecke, J., 1995. Stochastic Geometry and Its Applications, second ed. Wiley.

Thiessen, A.H., 1911. Precipitation averages for large areas. Monthly Weather Review 39, 1082-1084.

Wolfowitz, J., 1949. The distribution of plane angles of contact. Quarterly of Applied Mathematics 7, 117-120. 